\title{
Alimentos con calidad. Nuevas estrategias rurales para nuevos consumidores
}

\author{
Encarnación Aguilar Criado \\ Universidad de Sevilla \\ eaguilar@us.es \\ Santiago Amaya Corchuelo \\ Universidad de Cádiz \\ santiago.amaya@uca.es \\ Ignacio López Moreno \\ UAM, Lerma, México \\ ignacio.lopezmoreno@gmail.com
}

Resumen: Este texto profundiza en uno de los aspectos que caracteriza a la nueva ruralidad: la progresiva especialización de los espacios rurales en producción de calidad y el uso de marcas distintivas. Este tipo de estrategia de creación de valor añadido en los productos alimentarios ha sido una de las líneas prioritarias de los Programas de Desarrollo Rural en Europa desde los inicios del siglo xxi. Esta incesante proliferación de marcas de calidad de productos agroalimentarios denota la profunda transformación con que los espacios rurales han entrado en el nuevo siglo.

Palabras clave: nueva ruralidad; antropología; alimentación; indicaciones geográficas; Denominación de Origen Protegida.

Abstract: This text takes an in-depth look at one of the aspects that characterises the new rurality; that is the progressive specialisation of rural spaces in the production of quality foods and the use of distinctive quality labels. This means of creating added value for food products has been one the priority strategies of the Rural Development Programme in Europe since the beginning of the 21st century. The incessant proliferation of quality labels for food products indicates the profound transformation of rural spaces as they have entered the new century. Keywords: new rurality; anthropology; food; protected geographic indication; protected designation of origin. 


\section{Las marcas de calidad y sus paradojas}

Este texto profundiza en uno de los aspectos que caracteriza a la nueva ruralidad: la progresiva especialización de los espacios rurales en producción de calidad y el uso de marcas distintivas ${ }^{1}$. Este tipo de estrategia de creación de valor añadido en los productos alimentarios ha sido una de las líneas prioritarias de los Programas de Desarrollo Rural en Europa desde los inicios del siglo xxI. Esta incesante proliferación de marcas de calidad de productos agroalimentarios denota la profunda transformación con que los espacios rurales han entrado en el nuevo siglo. Nos habla de la transición que han recorrido estos territorios desde su supremacía agraria hacia la diversificación productiva, de la búsqueda de una economía de valor capaz de responder a los nuevos retos de la globalización (Cloke et alii, 2006).

Las marcas de calidad han sido objeto de diversos tipos de estudio. Por un lado, los que analizan el etiquetado como una estrategia económica de los productores, considerado como iniciativa interesante para reducir la sobreproducción, mejorar la competitividad de las áreas más pobres y dotar a los pequeños productores y emprendedores de una herramienta con que diferenciar sus productos y competir en los mercados globales (Knickel y Renting 2000; Miele y Pinducciu, 2001; Banks y Marsden, 2001; Pugliese 2001; Tregear et alii, 2007). En un sentido más amplio, otros estudios han insistido en la relación entre marcas de calidad y la Política Agrícola Común (PAC), demostrando el creciente apoyo que los Programas de Desarrollo Rural han tenido en la protección y revitalización de los productos regionales (Murdoch et alii, 2000). Esta línea de trabajo subraya la capacidad de activación territorial de estos productos, así como los beneficios generados para las comunidades donde se crean, tanto en el ámbito económico, como en el cultural y social.

Ambas aproximaciones se complementan con otras líneas de trabajo que relacionan el auge de las etiquetas con nuevas prácticas agrícolas y medioambientales, señalando su impacto positivo en la reducción de la contaminación y su

1 La investigación ha sido financiada por los proyectos: «La producción de calidad: nuevas estrategias rurales para nuevos consumidores», Ministerio de Educación y Ciencia, I+D (CSO2010-22074-C03-01), y «Las marcas de calidad en el mundo rural: nuevos retos para productores y consumidores», I+D (CSO201342468-P), Ministerio de Economía y Competitividad y Fondo Feder. Incentivos de Investigación del Programa Talentia (Junta de Andalucía). Grupo de Investigación TECUDE . P.A.I. SEJ-418). 
contribución a crear sistemas productivos sostenibles (Kaltoft, 1999; Marsden y Smith, 2005; Lozano, 2011).

Asumiendo las indudables ventajas comparativas que este tipo de especialización productiva ha supuesto para los territorios rurales, este texto pretender profundizar en algunos aspectos menos tratados por esta literatura científica, desvelando algunas contradicciones y conflictos igualmente inherentes a este tipo de iniciativas. Y ello porque, como trataremos de demostrar, los procesos de implantación de las marcas de calidad constituyen un fenómeno polivalente, en la medida que suponen tanto un espacio de concertación entre distintos productores e instituciones como un ámbito de expresión de poder y conflicto entre intereses de distintos signos. Suponen procesos sociales muy complejos, en los que los actores involucrados tienen que construir un proyecto colectivo, tendente a re-pensar y re-diseñar el producto que etiquetar (Marsden y Smith, 2005), lo que significa igualmente elaborar una normativa que lo defina y lo singularice.

Este proceso puede aumentar la cohesión y sinergia entre los actores del territorio, pero también puede excluir a aquellos que formaban parte del sistema previo de elaboración del producto - ya sea por incapacidad de asumir los costes de transformación o por disconformidad con el nuevo sistema- que, con la nueva legalidad se ven desposeídos del capital simbólico que su producto tenía al ser legalmente monopolizado por el nuevo sello.

Tal y como trataremos de demostrar, la creación de una marca de calidad conlleva la creación de un marco institucional y la elaboración de un cuerpo normativo que, a partir de entonces, regulará las características del producto. Una construcción restrictiva a la fuerza, que conllevará la selección de un conjunto de materias primas, manejos y técnicas preexistentes y sus límites territoriales, lo que determinará el conjunto de características consustanciales a la certificación de la nueva marca. Está claro que esta nueva situación limitará usos y manejos tradicionales y generará además nuevas relaciones de poder entre los actores implicados (Ménard, 1990; Wilkinson, 1997), y como veremos, marcará transiciones y cambios sociales en estos territorios rurales (López y Aguilar, 2015).

Este enfoque más crítico de la estrategia de la diferenciación de los productos rurales permite subrayar que los factores de inclusión y exclusión son consustanciales a los procesos de certificación de calidad, por cuanto conlle- 
van prohibición, apropiación y homogeneización de determinadas prácticas y de saberes locales, lo que implica también una pérdida de diversidad cultural (Bowen y de Master, 2011). El resultado es en realidad un nuevo producto, creado a partir de una eficiente combinación entre tecnología tradicional e innovaciones técnicas y sanitarias actuales. Un producto que, además, utiliza el discurso de la tradición y su anclaje territorial como parte sustancial de su marketing comercial.

Esta perspectiva permite, además, contemplar la viabilidad económica de estas estrategias de diferenciación a lo largo de la cadena alimentaria. Hemos de tener en cuenta que la construcción del proceso supone la inversión de capital, tiempo y energía por parte de todos los actores involucrados. Dada la creciente jerarquización entre productores y distribuidores de las actuales cadenas alimentarias (Massot, 2015), veremos como el incremento del coste final del producto determinará la diversificación de estrategia de los pequeños productores.

\section{Calidad y nuevos territorios rurales}

El aumento de las marcas de calidad constituye un fenómeno ligado tanto a las nuevas funciones de los espacios rurales como a recientes demandas de los consumidores de las sociedades posindustriales (Lash y Urry, 1998). Es evidente que han constituido uno de los principales objetivos de los Programas de Desarrollo Rural en Europa, en la medida en que son acciones demostrativas del enfoque territorial que se incorporaría a la reforma de la PAC de comienzos del año 2000 (O’Connor, 2006).

Este nuevo concepto del territorio, que de mero soporte de actividades se convierte en un recurso para el desarrollo (Esparcia y Escribano, 2012), resulta crucial para entender la potencialidad que poseen estos productos con anclaje territorial para los Programas de Desarrollo Rural (Aguilar, 2015). Por esa razón han sido el centro de numerosas iniciativas lideradas institucionalmente desde los Grupos de Desarrollo Local (GDR) con fuentes de financiación europea (López et alii, 2015).

Proteger los productos locales cumplía plenamente con los objetivos de unos proyectos de desarrollo tendentes a diversificar las economías rurales, apoyando proyectos ligados a los valores tangibles e intangibles de la cultura lo- 
cal, capaces de generar externalidades positivas y de contribuir al mantenimiento de la estructura demográfica y de los mercados de trabajo (Rubio, 2010). Significaba, además, cumplir con las nuevas directrices de la PAC, en tanto que fomentaba la biodiversidad y la reducción de los agentes contaminantes de la agricultura intensiva. En definitiva, producir alimentos acordes con un modelo de consumo que privilegia los productos diferenciales, especialmente aquellos que incluyen un elevado grado de seguridad.

No olvidemos que esta nueva tendencia del consumo es la respuesta ciudadana a recientes alarmas alimentarias, impulsora de normativas sobre la trazabilidad de los alimentos (Espeitx, 1996). En un entorno actual de sobreabundancia alimentaria (Guidonet, 2010), gobernado por monopolios agroindustriales que han culminado la desconexión entre campo y plato, los productos de calidad se erigen como garantes de seguridad alimentaria, dotados además del valor añadido que le proporciona su vinculación con un territorio concreto. De esta forma, los elementos consustanciales de una particular ecología, tradición e historia, se incorporan como elementos distintivos de esos productos locales (Lozano y Aguilar, 2010), y es a partir de esa transferencia de significados sociales como logran sus características diferenciales de cara a mercados cada vez más globales (Costanigro et alii, 2009).

En este contexto que ha hecho proliferar las marcas de calidad destacan, al menos en el caso europeo, la Denominación de Origen Protegida (DOP), la Indicación Geográfica Protegida (IGP), o el sello de Agricultura Ecológica, como certificaciones de mayor nivel de control y reconocimiento europeo. A partir de ellas se han ido creando un entramado de otras muchas de menor rango, sellos territoriales de distinto signo que tratan de subrayar la vinculación de estos productos con una zona protegida (marca parque natural), con una comarca o región de procedencia (marca territorial), por solo citar algunos de los casos más conocidos.

Para profundizar empíricamente en los aspectos teóricos hasta aquí planteados hemos seleccionado dos productos que mantienen un importante anclaje territorial y que forman parte de la gama de alimentos gourmet. Nos referimos a los quesos de la Sierra de Cádiz (Andalucía), y al jamón ibérico de bellota, en este caso certificado con cuatro DOP, dos de ellas ubicadas en Andalucía y las dos restantes en Extremadura y Castilla, respectivamente. Constituyen parte de los resultados de una investigaciie una investigacis productores.sentan algu- 
nasde las cuestiones hasta aqui ón de cinco años de duración (de 2009 a 2013) realizada en distintas zonas de Europa y Brasil, con el objetivo de comparar la situación de este proceso en dos zonas geopolíticas distintas ${ }^{2}$.

El estudio se ha basado en una metodología de tipo cualitativo, a partir de un trabajo de campo en las distintas zonas, para la que se confeccionó una muestra de actores relevantes clasificados según su distinta posición y ámbitos de poder en las iniciativas estudiadas: productores, técnicos pertenecientes a las distintas entidades vinculadas al desarrollo territorial en sus diversos ámbitos: regionales, provinciales o locales, representantes políticos y asociaciones locales de distinto signo, tanto las surgidas en relación con la puesta en marcha de este tipo de iniciativas económicas, o bien como apoyo a las mismas (tabla 1). A esta muestra se le realizó una entrevista tipo semidirigida estructurada.

\section{Tabla 1. Distribución de entrevistas según tipología y unidad de observación}

\begin{tabular}{|c|c|c|c|c|c|}
\cline { 2 - 6 } \multicolumn{1}{c|}{} & Productores & Técnicos & Políticos & Asociaciones & Total \\
\hline $\begin{array}{c}\text { Jamón ibérico } \\
\text { de bellota }\end{array}$ & 18 & 13 & 1 & 1 & 32 \\
\hline $\begin{array}{c}\text { Quesos Sierra } \\
\text { de Cádiz }\end{array}$ & 44 & 13 & 1 & 5 & 63 \\
\hline Total & 62 & 26 & 1 & 6 & 95 \\
\hline
\end{tabular}

La selección de estos dos productos nos va a permitir profundizar en los dos temas centrales de este trabajo: los procesos de transición, cambios y conflictos vinculados a la construcción de una marca de calidad, y su viabilidad como estrategia de los pequeños productores rurales.

\footnotetext{
2 La muestra total está constituida por trescientas entrevistas, y se centró en las siguientes marcas de productos y zona: DOP de Jamones Ibéricos de Extremadura, Valle de los Pedroches y Jabugo; DOP de Quesos de Asturias; Marca de Calidad de Queso de Sierra de Cádiz; Marca Territorial y Echt Texels Lamvless (Holanda); IGP de Valle de Vihnedos y Carme de la Pampa (Brasil); Marca Territorial de la Comarca de Guadalteba (España), y el Pays Gorges, Causses, Cevennes (Francia), así como la producción con marca de Agricultura Integrada y Ecológica de Arroz en la zona de Isla Mayor en Sevilla, y la DOP de Arroz Litoral Norte y Arroz Ecológico de Taim (Brasil). Para el desarrollo del proyecto se contó con la colaboración de un equipo de investigadores brasileños y la financiación del Programa Hispano-Brasileño de Cooperación Interuniversitaria. Ministerio de Ciencia e Innovación y CAPES. (PHB2008-0043-PC).
} 


\section{La construcción institucional de las marcas}

La zona de producción quesera de la Sierra de Cádiz está mayoritariamente enclavada en el Parque Natural de la Sierra de Grazalema³. En este territorio, de tradición ganadera y una importante especialización en turismo rural y de naturaleza, se ubican la mayoría de los actores involucrados en una pujante industria quesera en la actualidad.

El sistema tradicional de producción de queso curado formaba parte de las prácticas productivas locales desde largo tiempo atrás, una producción ligada a las prácticas domésticas de las familias de pastores de estos espacios de montaña. La elaboración casera de quesos constituía una de tantas estrategias asociadas a la lógica doméstica de estas familias de pastores, que igualmente vivían de la venta de la carne del cordero o de la transformación de la lana y fabricación artesanal de mantas (López y Aguilar, 2013). El caso de la producción quesera es un ejemplo paradigmático del proceso de transición de una sociedad rural tradicional a la nueva ruralidad. Ha tenido dos momentos muy claros en los últimos cincuenta años, y fue la entrada de España en la entonces CEE (Comunidad Económica Europea) su punto de inflexión. Dicha entrada supuso la implementación de un nuevo conjunto de regulaciones para la industria láctea, que no tenía en cuenta las escalas o contextos productivos. Los nuevos requisitos supusieron, en la práctica, la ilegalización de la producción tradicional de queso en Sierra de Grazalema.

La lógica de este sistema doméstico de producción quesera se caracterizaba por la división sexual del trabajo: el hombre pastor y la mujer quesera. El manejo tradicional, que aún hoy en día se mantiene en muchas fincas, se basa en una forma de alimentación que busca la autosuficiencia del animal y limita el tamaño de los rebaños a la capacidad de carga del terreno y de manejo del pastor, lo que supone un total de entre cien y doscientas ovejas en extensivo. Este sistema tradicional, que se realizaba en las fincas ubicadas fuera del casco urbano, se vio afectado por un nuevo orden legal. Primero en materia de sanidad, marcado por la legislación del Consejo Europeo, que en 1992 estableció las normas sanitarias aplicables a la producción y comercialización de leche cruda,

3 La Sierra de Cádiz es un área montañosa situada al suroeste de Andalucía; está compuesta por 19 municipios, tiene una extensión total de 1998 km² y 116792 habitantes. Su definición territorial actual corresponde al proceso de comarcalización para la implementación de las Iniciativas Leader I. De hecho, el GDR de la Sierra de Cádiz fue uno de los primeros en crearse en Europa en el año 1991. 
leche tratada térmicamente y productos lácteos. A estas primeras directrices se sumaron las emanadas de la nueva figura de protección ambiental que la zona adquiere también en esa misma década, con la creación del Parque Natural Siera de Grazalema, lo que supuso la muerte virtual de unas prácticas y manejos técnicos legendarios, que han sido centrales en la reproducción ecológica, social y cultural de este territorio.

Fue precisamente el peso de ese saber hacer tradicional el que determinó la transición hacia el sistema de mercado de esta elaboración doméstica del queso. En esa misma década de los noventa, y al calor de los Programas de Desarrollo Rural, europeos, surgió la industria quesera en la zona mediante inversiones en las primeras fábricas, ya dotadas con unas instalaciones que se adaptaban a las nuevas exigencias técnicas de salubridad y seguridad alimentarias europeas (López, 2014).

Cuando abrimos la fábrica, nosotros estábamos pensando en el desarrollo de la comarca, porque si no, la oveja merina de Grazalema se pierde, porque nadie les compraría la leche. Antes de abrir la fábrica, el queso lo hacían los pastores, pero la normativa nueva no encajaba; para hacer queso tenían que tener unas condiciones higiénicas y sanitarias que no podían cumplir. Aquí había una tradición familiar, pero había que adaptarla a la nueva normativa. (Propietario de quesería El Payoyo, Villaluenga del Rosario, Cádiz)

Nacieron así las dos nuevas industrias locales: El Bosqueño y la Quesería El Payoyo, ambas con el importante apoyo institucional del GDR de la zona y la subvenci,con la necesariar IIIdLeaII. çoe Acciçon Local de la zona y la subvenciuna reg tratan de subrayar la vinculación de las Iniciativas Leader. El éxito de estas primeras fábricas determinaría un proceso imparable de creciente apertura de nuevas queserías por todo el territorio hasta alcanzar las veintitrés actuales, y un aumento continuado de las cabezas hasta llegar al $50 \%$ más que en 1996.

En este contexto de creciente éxito de un producto local en el mercado que contenía todos los elementos de singularidad que venimos comentando, se iniciaron las acciones para crear una DOP de Quesos de Oveja de la Sierra de Cádiz en 2002, con la necesaria concertación de todos los actores locales, tanto institucionales como productores ${ }^{4}$. Fue el mismo GDR de la Sierra de Cádiz

4 La Comisión Impulsora de la DOP Quesos de la Sierra de Cádiz se formó con la participación de representantes de la Diputación de Cádiz, la Oficina Comarcal Agraria de la Sierra de Cádiz, el GDR de la Sierra 
el que lideró el proceso, y quizás fue esa excesiva dependencia institucional la causante de que hasta el día de hoy se trate de una iniciativa inconclusa, que ni siquiera ha conseguido llegar a su tramitación ante el Ministerio de Agricultura español. Las necesarias, pero también excesivas, trabas burocráticas y niveles de regulación que exigen la DOP explican el desinterés de los mismos productores por completar este largo proceso.

Lo más significativo de esta transición es que no podemos hablar de fracaso, sino todo lo contrario, porque el intento de creación de la $\mathrm{DOP}^{5}$ ha generado sinergias entre los productores y transformadores locales que, mediante reuniones, talleres y visitas mutuas han terminado por aceptar la complejidad del proceso productivo y la apuesta decisiva por la calidad del mismo.

A día de hoy, la apuesta por la distinción y la calidad es clara entre los transformadores. Las excelencias de su producto, ligado a las características especiales de su territorio, están asumidas por productores y compradores, y el queso no necesita de más signos de calidad que el que otorga a un entorno tan peculiar como el del Parque Natural de la Sierra de Grazalema, que atrae a turistas que buscan la especificidad de sus artesanías y su gastronomía, además de su paisaje. En este contexto, ya de por sí tan singularizado, lo que no les sirve a estos nuevos empresarios es la figura de una DOP, dadas las estrictas regulaciones que conllevan y el coste excesivo que les supondría. Les basta con una marca territorial que ampara un producto gourmet, ganador de premios nacionales e internacionales y bien posicionado en los mercados.

Es verdad que esta transición ha producido también rupturas y exclusiones, algunas de ellas muy importantes, como la desaparición de la mujer como productora de queso. Las queserías industriales han sido abiertas en su mayoría por hombres empresarios, lo que ha supuesto la ocupación de un espacio tradicionalmente femenino, como era la transformación y venta de la leche en queso a una escala doméstica. También han sido muchas las familias de pastores que no han podido integrarse en el nuevo proceso de transformación y se han visto abocadas al cambio de actividad. Con ellas también han desaparecido rebaños y formas de producción que nunca más podrán ser rescatados.

de Cádiz, la Mancomunidad de Municipios de la Sierra de Cádiz, la Asociación de Criadores de la Raza Ovina Merina de Grazalema, la Asociación de Criadores de Raza Caprina Payoya y varios queseros de la zona.

5 Dentro del proceso de intento de crear la DOP destaca la creación de una Feria Artesanal del Queso de la Sierra de Cádiz, que ya cuenta con ocho ediciones y reúne a más de treinta empresas entre productores locales y foráneos de la Sierra de Cádiz. 
Pero también es verdad que la interacción y el aprendizaje realizado durante los diez años que se invirtieron en el proyecto de obtener una DOP han supuesto la ruptura de la visión localista y sectorial de los actores implicados, pasando a percibir la economía en clave territorial. El sector quesero se ha convertido en un nuevo pilar que articula la economía del territorio, y tanto los pastores como los queseros participan de proyectos e iniciativas territoriales fuera de su sector. Las claves del éxito de este caso residen en un ajustado equilibrio entre tradición e innovación. En unas innovaciones técnicas introducidas para cumplir con los nuevos requerimientos de salubridad y seguridad alimentaria, junto a un eficiente manejo de los pastos de un entorno singular que alimentan a las dos razas autóctonas: la oveja merina grazalemeña y la cabra payoya.

\section{Calidad, mercado y confianza}

La actual industria del jamón ibérico de bellota aparece ligada al sistema de explotación intensiva tradicional de un ecosistema característico del bosque mediterráneo. Formaba parte, al igual que los quesos de Sierra de Cádiz, de un sistema de de un sistema de explotación campesina tradicional. Un sistema que sufrió una primera trasformación hacia su industrialización a partir de los años ochenta, y que posteriormente ha conseguido con éxito su reconocimiento como DOP. En la actualidad existen cuatro DOP de jamón ibérico: Jabugo, Valle de los Pedroches, Dehesa de Extremadura y Guijuelo, que coinciden con aquellos territorios con presencia de bosque de dehesa ${ }^{6}$ de Andalucía, Extremadura y Castilla, respectivamente.

La particularidad de este tipo de jamones radica en el binomio socialmente extendido ibérico-bellota, o lo que es igual, ibérico-dehesa, que subraya el vínculo entre las producciones de cerdo ibérico engordado con bellotas, con su ecosistema singular. Dicho binomio encierra la máxima socialmente construida y compartida de que este producto está indisolublemente unido a un territorio antropizado, fruto de un secular sistema de explotación, donde predomina una tipología de manejos ganaderos que constituyen un modo de gestión de un territorio basado en prácticas agronómicas tradicionales. La centralidad de estas prácticas reside en el aprovechamiento de la bellota para la alimentación,

6 La dehesa es un tipo de bosque mediterráneo compuesto, sobre todo, de encinas y alcornoques en grado variable de densidad, y de pastos herbáceos, con un tradicional sistema de aprovechamiento agrosilvopastoril en una interacción generalmente sostenible mediante prácticas y conocimientos agrícolas y ganaderos. 
engorde y cría del cerdo de raza ibérica, a partir de un sistema denominado montanera, que se corresponde con el periodo de maduración de la bellota (de noviembre a febrero) en que el ganadero reparte a los animales por la explotación (Amaya, 2014).

La tradición en las producciones del cerdo ibérico nos remite a la primera mitad del siglo pasado, cuando los procesos tradicionales de elaboración (domésticos y protoindustriales) de los derivados del cerdo ibérico estaban escasamente mecanizados. Se utilizaban materias primas de cierta calidad (carnes, grasas, sal, pimentón y ajo, sobre todo), características meteorológicas concretas (vientos, temperatura y humedad) y una especialización productiva basada en un profundísimo conocimiento local y control de todas estas variables (Amaya y Aguilar, 2012). El factor humano, su pericia, habilidades técnicas y conocimientos, eran los que verdaderamente determinaban estas producciones.

Este sistema tradicional de explotación, hoy definido como sostenible, se basa en el adecuado equilibrio entre la carga vegetal y la animal, que otorga unas cualidades únicas al jamón de bellota y lo convierte en uno de los productos gourmet por excelencia en España, valorado y apreciado por igual a nivel nacional e internacional. Desde estas bases tradicionales se ha producido la transición en la actualidad a una importante agroindustria, de indudable anclaje territorial, que explica la existencia misma de las distintas DOP ya mencionadas.

Alcanzar la certificación de este sello de calidad supone someter el proceso de producción a los estrictos sistemas de regulación establecidos para su reconocimiento. Los reglamentos de los Consejos Reguladores supervisan la calidad del producto a partir de un complejo sistema de certificación que permite garantizar su trazabilidad. En este caso, estipulan las características de la raza del cerdo, la edad de los animales, su alimentación, el periodo de curación de sus derivados y los métodos de elaboración permitidos. Se produce así la mencionada selección de determinadas características del modelo tradicional de producción del cerdo ibérico de montanera, a partir de los elementos que se adecuan a los parámetros reglamentarios de la Unión Europea para las DOP. De nuevo, se establece esa precisa combinación entre tradición — de usos, manejos y saberes locales - con innovación y requerimientos tecnológicos - de higiene y salubridad - en la que radica la construcción de la calidad, que ahora se oferta bajo la fórmula de marca protegida (Amaya y Aguilar, 2013). 
La cuestión es que estos estrictos requisitos de calidad que fijan las DOP terminan por hacer inviable la producción para muchos productores:

La DOP es un gasto tremendo. La semana pasada estuvieron marcando unos para la DOP y ante una pequeña traba, les dije: «Ponme lo que quieras, menos problemas», porque yo, para vender un jamón de bellota, no me hace falta que lleve ninguna etiqueta de DOP.

[...] los he tenido refrigerados más de los noventa días, y los técnicos de la DOP me ven que estoy sacando jamones del secadero. Es cierto que el secadero se encuentra a humedad libre, pero a temperatura máxima de $12^{\circ} \mathrm{C}$, porque a partir de los $11^{\circ}$ los jamones sufren. Pero con $12^{\circ}$ los jamones están completamente suaves, es cuando empiezan a sudar. Bueno, pues te recrimina porque eso no es correcto. (Ganadero e industrial de la DOP Valle de los Pedroches, Córdoba)

Estar o no dentro de la certificación de DOP se convierte así en una estrategia que los mismos productores eligen en relación con la capacidad y tamaño productivo de su industria, a los precios de la materia prima y a la demanda del mercado. De ahí que en 2011 y según los datos del Ministerio de Agricultura, Alimentación y Medio Ambiente, apenas un $10 \%$ de la producción de jamones ibéricos se acogieran a alguno de estos cuatro sellos existentes. Lo que sucede es que cada campaña, y en función de los costes y beneficios, de los precios del producto en el mercado, cada productor decide qué número de cabezas destinar a su certificación. Lo cierto es que la DOP no supone una mayor calidad de un producto que, como hemos señalado, sigue requiriendo del singular sistema de elaboración y de saber hacer local tradicional ya mencionado, sobre los que hoy se construye la trazabilidad de la marca que lo liga a la cultura y a la identidad de estos territorios.

Por eso, más allá del valor que las DOP adquieren en los mercados más globales, en la escala local funcionan otros parámetros de calidad, basados en sistemas menos formalizados y propios de la economía de proximidad, como el conocimiento y la confianza entre comprador y productor. $Y$ este es uno de los criterios que el productor maneja a la hora de elegir el tipo de estrategias que seguir en cuanto al porcentaje de cerdos a certificar o incluso de su retirada de la DOP, una decisión que ha sido demasiado común, especialmente entre los pequeños productores, en los actuales momentos de crisis. 


\section{Conclusiones}

Como señalábamos al principio, en este texto no hemos tratado de negar las sinergias positivas que las marcas de calidad han supuesto para los pequeños productores, frente al poder de las multinacionales de la agroalimentación de productos industrializados; creemos que este tipo de sinergias positivas que profundizan en la relación entre nueva ruralidad y desarrollo territorial ya ha sido suficientemente estudiada, de ahí que hayamos querido desvelar aquellos aspectos menos positivos que estas etiquetas también contienen.

El análisis comparativo de nuestra investigación nos ha demostrado que existen grandes diferencias en los costes de transacción en las diferentes marcas. El rango más alto en los mercados lo ocupan las DOP, por eso son, sin lugar a dudas, las que mayores costes acarrean a los productores, comenzando por el largo y complejo proceso que conllevan su reglamentación y reconocimiento a nivel europeo, y siguiendo por la rigidez de sus requisitos y restricciones técnicas que a los productores les supone. Son estos obstáculos los que hacen que muchos actores pierdan interés en la solicitud de una certificación como la DOP.

Temporalmente hablando, la obtención de una DOP puede dilatarse hasta unos diez años. Este trámite supone un esfuerzo de concertación social de las partes implicadas, marcado por la participación en reuniones, actividades y debates entre productores de distinto tipo y con intereses no siempre coincidentes, lo que determina el grado de interés o de implicación de muchos y el esfuerzo personal, incluso de inversión de tiempo en continuas reuniones, que muchos no quieren o no pueden realizar. La construcción del consenso es un proceso delicado, y sin la participación de un intermediario imparcial, resulta muy difícil que las dinámicas de poder no condicionen o limiten los resultados.

Es verdad que las etiquetas de calidad suponen un incremento del coste económico en cada paso de la cadena, lo que, en teoría, supone que la rentabilidad final será también mayor. Pero lo que sucede a menudo es que este aumento de coste y de valor añadido no se reparte de manera equilibrada entre las diferentes partes de la cadena agroalimentaria, entre grandes y pequeños productores y entre elaboradores y distribuidores. A partir de ahí, aparecerán estrategias alternativas, tal y como hemos visto en los dos casos estudiados. 
En el caso del jamón ibérico, los mismos productores de las DOP diversifican sus estrategias entre partidas con DOP para mercados externos, y los de mercado local, amparados por certificaciones menos formalizadas. En el caso de Quesos de Sierra de Cádiz, los elaboradores eligieron marcas de menos nivel para sus productos, cuyo anclaje territorial era ya garante de calidad.

La función del sello de calidad está, por tanto, relacionada con la distancia geográfica entre productor y consumidor. Allí donde hay proximidad entre ambos, actúan otros parámetros menos formalizados que tienen que ver con la cultura local. El conocimiento y la confianza entre productor y consumidor se consagran así en los elementos fundamentales que ampara la calidad del producto y garantiza su venta de cara al consumidor. Un mecanismo menos formalizado al que se acude de manera creciente en los actuales momentos de crisis económica.

\section{Bibliografía}

Aguilar, E. (2014). «Los nuevos escenarios rurales: de la agricultura a la multifuncionalidad». Endoxa, 33: 73-98.

Amaya, S. (2014). «Una lectura de la agricultura familiar en áreas de dehesa», Agricultura familiar en España. Fundación de Estudios Rurales. Anuario 2014: 276-282.

Amaya, S. y Aguilar, E. (2013). «La construcción de la calidad alimentaria: tradición, innovación y poder en las DOP del jamón ibérico en España». Revista de Economía Agricola, 59 (2):39- 52.

Banks, J. y Marsden, T. (2001). "The Nature of Rural Development: the Organic Potential». Journal of Environmental Policy and Planning, 3 (2): 103-121.

Bowen, S. y de Master, K. (2011)+ «New rural livelihoods or museums of production? Quality food initiatives in practice». Journal of Rural Studies, 27: 73-82.

Cloke, P. T.; Marsden, T., y Mooney, P. H. (eds.) (2006). Handbook of Rural Studies, London: Sage.

Espeitx, E. (1996). «Los nuevos consumidores o las nuevas relaciones entre campo y ciudad a través de los productos de la tierra». Agricultura y Sociedad, 80-81: 83-116. 
Esparcia, J. y Escribano, J. (2012). «La dimensión territorial en la programación». Anales de Geografía, 32 (2): 227-252.

Díaz, C.y Gómez, C. (2008). Alimentación, consumo y salud. Barcelona: Obra Social. Fundación La Caixa.

Guidonet, A. (2010). ¿Miedo a comer? Crisis alimentaria en contexto de abundancia. Barcelona: Icaria.

Kaltoft, P. (1999). «Values about Nature in Organic Farming Practice and Knowledge», Sociologia Ruralis, 39 (1):39-53.

Knickel, K. y Renting, H. (2000)* «Methodological and Conceptual Issues in the Study of Multifunctionality and Rural Development». Sociologia Ruralis, 40 (4): 512-528.

Lash S. y Urry, J. (1998). Economías de Signos y Espacios. Buenos Aires: Amorrutu.

López, I. (2014). Labelling the origin food products: Towards sustainable territorial development? Wageningen: Ponsen $\&$ Looijen.

López, I. y Aguilar, E. (2013). «La nueva economía rural europea. Especialización territorial de calidad en la Isla de Texel y la Sierra de Cádiz». Gazeta de Antropología, 29(2), artículo 04.

- (2015). «Resiliencia y cambios del sistema productivo en la agricultura familiar de la Nueva Ruralidad europea: el caso del queso de oveja en la Sierra de Cádiz (España)». Tessituras, 4 (2)ः 242-268.

López, I.; Aguilar, E.; Lozano, C., y Pérez, A. (2015). "Quality labels and institutional density in the agro-food sector: the case of Andalusia (Spain)». Spanish Journal of Rural Development, 3 (VI): 9-19.

Lozano, C. (2011). El sabor de la naturaleza: agricultura ecológica en Parques Naturales andaluces. Sevilla: Fundación Blas Infante.

Lozano, C. y Aguilar, E. (2010). «Natural, tradicional y de la tierra. La promoción de la calidad agroalimentaria en los nuevos espacios rurales andaluces». En M. Soler y C. Guerra (eds.), Patrimonio cultural en la nueva ruralidad andaluza. Sevilla: Instituto Andaluz del Patrimonio Histórico, 126-139.

Lozano, C. y Aguilar, E. (2012). «Territorialising organic production: collective actions and public policies in Andalusia». Sviluppo Locale, XV: 45-66. 
Marsden, T., y Smith, E., (2005). «Ecological Entrepreneurship, Sustainable Development in Local Communities through Quality Food Production and Local Branding». GeoForum, 36: 440-451.

Massot, A. (2015). «De la política de mercados agrarios a la política de la cadena alimentaria en la PAC 2020». Fundación de Estudios Rurales, Anuario 2015: 32-42.

Ménard, C. (1990). L'économie des organisations. Paris: La Découverte

Miele, M. y Pinducciu, D. (2001). «A Market for Nature: Linking the Production and Consumption of Organics in Tuscany» Journal of Environmental Policy and Planning, 3 (2): 149-162.

Murdoch, J.; Marsden, T., y Banks, J. (2000). "Quality, Nature, and Embeddedness: some Theoretical Considerations in the Context of the Food Sector» Economic Geography, 76 (2): 107-125.

O'Connor, D. (ed.) (2006). Driving rural development: policy and practice in seven EU countries. Assen: Uitgeverij Van Gorcum.

Pugliese, P. (2001). «Organic Farming and Sustainable Rural Development: A Multifaceted and Promising Convergence». Sociologia Ruralis, 41 (1): 112-130.

Rubio, P. (2010). «Modelización de los cambios y evolución reciente del sistema rural español». AGER, 54:203-235.

Treagear, A.; Arfini, F*; Belletti, G., y Mascerotti, A. (2007). «Regional Foods and Rural Development: The Role of Product Qualification», Journal of Rural Studies, 23 (1): 12-22.

Wilkinson, J. (1997), «A New Paradigm for Economic Analysis?». Economy and Society, 26(3):305-39. 